\title{
¿Por qué no llenar la botella?: Un estudio sobre el concepto de capacidad con niños escolarizados ${ }^{1}$
}

\author{
Why not fill the bottle?: A study of the concept \\ of capacity with school children
}

\section{Por que não encher a garrafa: Um estudo sobre o conceito de capacidade com as crianças da escola}

Recibido: mayo de 2013

Aceptado: agosto de 2013
Mg.Erika Rosana Calambás Córdoba²

\section{Resumen}

Verificando la indiferenciación que tanto autores como libros de texto presentan de los conceptos capacidad y volumen, se construyeron cinco situaciones, las cuales fueron presentadas a 18 niñas y niños, de 11-12 años de edad y tres grupos poblacionales (Campesinos, Indígenas e Hijos de Asalariados) de la Institución Educativa Agropecuaria la Capilla- Cajibío (Cauca). Para dichas situaciones se utilizaron diversos recipientes. En el trabajo se identificaron y se jerarquizaron estrategias que los niños desarrollan en la solución de las situaciones y se levantaron hipótesis frente a las posibles influencias que el contexto social ejerce sobre las estrategias utilizadas por las niñas y los niños en la construcción de las soluciones.

Palabras clave: Capacidad; Volumen; Situaciones; Estrategias; Alumno; Diversidad, Aspectos socioeconómicos.

\section{Abstract}

Verifying that both authors indifferentiation textbooks present concepts and volume capacity were built five situations, which were presented to 18 children, 11-12 years old and 3 populations (Peasant, Indigenous and Sons of Employees) of School Chapel-Cajibío Agropecuaria (Cauca). Such situations are used various containers. The paper identified and nested children develop strategies to solve situations and rose against the possible hypothesis that the social influences exerted on the strategies used by girls and boys in the construction of solutions.

Keywords: Capacity, Volume, Situations, Strategies, Student, Diversity, Socioeconomic aspects.

\section{Resumo}

Verificando que os dois autores indiferenciação livros didáticos atuais conceitos e capacidade de volume foram construídas cinco situações, que foram apresentados para 18 crianças, 11-12 anos de idade e três populações (camponês, indígena e Sons de funcionários) da Escola Chapel-Cajibío Agropecuaria (Cauca). Tais situações são usados vários recipientes. O papel

1 Artículo de Investigación

2 Institución Educativa Agropecuaria la Capilla- Cajibío (Cauca). Docente. Contacto: ercalambas@unicauca.edu.co 
identificados e crianças aninhadas desenvolver estratégias para resolver situações e se levantaram contra a possível hipótese de que as influências sociais exercidas sobre as estratégias utilizadas pelos meninos e meninas na construção de soluções.

Palavras-chave: capacidade, volume, situações, estratégias, Estudante, Diversidade, aspectos socioeconômicos

\section{Presentación del problema}

Esta ponencia tiene como marco de referencia el proyecto de investigación denominado "El concepto de capacidad en niños: un estudio de estrategias escolares para su construcción" (Calambás, 2011), en el cual se planteó como pregunta de investigación: ¿Qué estrategias desarrollan los niños al resolver situaciones problema que involucran el concepto matemático de capacidad y sirven de apoyo para el diseño de secuencias didácticas? Y cuyo objetivo fue el de identificar las estrategias que desarrollan los niños al resolver situaciones problema que involucran el concepto de capacidad.

Entre algunas reflexiones planteadas en el trabajo está el hecho de que en muchas ocasiones, los conceptos de volumen y capacidad se confunden. Es frecuente que ambos términos se utilicen como sinónimos. No es extraño escuchar, o encontrar en libros de texto, expresiones tales como: "calcule el volumen del balde" o "esa tasa tiene más volumen que esta otra", cuando en realidad, lo que se quiere es que el estudiante obtenga la capacidad de un balde o comparare la capacidad de dos tasas (Chamorro, 2003). Esto llevó a la construcción de una definición para el concepto de capacidad que dejara clara la diferencia que se establece entre el volumen y la capacidad, de la cual se identificaron los elementos necesarios para la construcción de las situaciones.

\section{Marco de Referencia Conceptual}

Para procurar dar cuenta del objetivo establecido, se propuso el análisis y discusión de las situaciones desde la perspectiva de Vergnaud(1982; 1990; 2006), quien plantea que el concepto de situación "se limitará al sentido que le da habitualmente el psicólogo: los procesos cognitivos y las respuestas del sujeto son función de las situaciones a las cuales son confrontados" (Vergnaud G., 1990, p.10).

Las soluciones aplicadas por los niños fueron denominadas estrategias, las cuales se consideraron como "toda secuencia de procedimientos, susceptibles de ser repetidos y transferidos a otras situaciones, que constituyen los medios para alcanzar el fin hacia el que tiende el sujeto" (Inhelder, 1978, p.7).

Por otra parte, con la revisión de antecedentes se pudo establecer que existe escasa documentación acerca del concepto de capacidad y poca precisión en las definiciones, lo que exigió la construcción de una definición para el concepto de capacidad, así: "Todo objeto que tiene como característica poder contener sustancias u objetos se denomina recipiente, y el máximo volumen que puede contener, sin sobrepasar los límites del mismo, se llama capacidad" (Calambás, 2011, p.57).

\section{Metodología}

Para el proyecto de investigación se determinó como método el enfoque de investigación cualitativo, construyéndose cinco situaciones, las cuales fueron aplicadas a 18 niños ( 9 mujeres y 9 hombres) entre los 11 y 12 años. Utilizándose como metodología la entrevista clínica (Poussin, 2005). Adicionalmente se tuvo en cuenta la diversidad de la población, estableciéndose tres grupos: indígenas, campesinos e hijos de asalariados.

A partir de la definición de capacidad, se estableció que se encuentra ligado al de recipiente; es por ello que en el desarrollo de las situaciones se utilizaron recipientes con formas, contenidos y espesores variados. 
Con respecto a las relaciones establecidas en las situaciones se identificaron estrategias y subestrategias, las cuales fueron organizadas según el grado de complejidad. En las situaciones se identificaron variables, como la movilidad del recipiente, que surgen del contexto, permitiendo levantar algunas hipótesis frente a las posibles influencias que el contexto social ejerce sobre las estrategias utilizadas por los niños en la construcción de las soluciones.

\section{Resultados Obtenidos}

Situaciones problema. A partir de lo considerado por González \& Florez (2001, p.2), con respecto a la capacidad, se desarrollaron actividades con los niños, cuyos resultados permitieron establecer, relaciones entre capacidad y contenido, capacidad y forma, capacidad y espesor, y Transformaciones.

Para la situación Capacidad y Contenido se utilizaron botellas con contenidos, niveles y líquidos distintos, con lo cual se buscó establecer la relación entre la capacidad y el contenido. Inicialmente se le presenta al niño dos botellas comerciales idénticas, una vacía y la otra llena y sellada y se le pide que llene la botella vacía hasta alcanzar su máximo contenido. Luego se le solicita que compare su resultado con el contenido comercial de la botella (botella sellada).

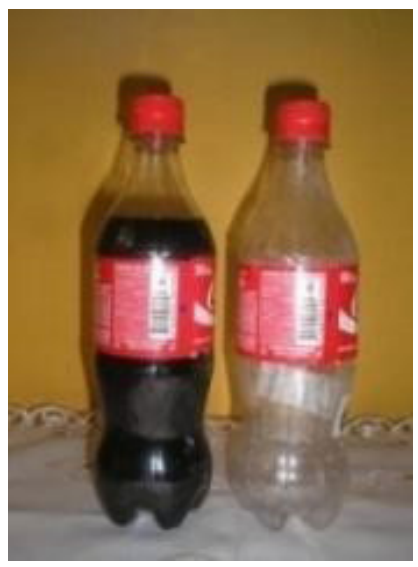

Estrategias. Las niñas y los niños utilizaron el tamaño como elemento de referencia para establecer orden, según Piaget \& Szeminska (1967) "el tamaño es la primera variable intuitiva que reconocen los niños al realizar comparaciones y tiene mayor presencia en los primeros años de escolaridad" (pp. 282-283). En este caso, las estrategias evidencian la relación entre el tamaño y el contenido del recipiente, al considerar que a mayor tamaño se corresponde un mayor contenido.

Se evidenció, además, que los niños hacen comparaciones con recipientes cuyas alturas son similares, pero cuando esto cambia se les dificulta establecer comparaciones, sobretodo identificar la conservación de la cantidad de líquido como lo plantea Piaget \& Szeminska (1967) "multiplicación de la relaciones ancho por alto donde el contenido en un recipiente bajo y ancho, vertido en otro alto y estrecho conserva su cantidad" (p.283).

Comentarios. acerca de posibles influencias socioculturales. A partir de la selección de estrategias y subestrategias se elaboraron cuadros como el siguiente:

Tabla 1

\begin{tabular}{|c|c|c|c|c|c|}
\hline ESTRATEGIA & SUBESTRATEGIA & C & I & HA & Total \\
\hline $\begin{array}{c}\text { Asignar el } \\
\text { contendido } \\
\text { como capacidad }\end{array}$ & $\begin{array}{c}\text { 1. Llenar hasta la } \\
\text { mitad del tarro o } \\
\text { muy cerca de ella. }\end{array}$ & $\begin{array}{c}50 \\
\%\end{array}$ & $\begin{array}{c}66.7 \\
\%\end{array}$ & $50 \%$ & 55.56 \\
\hline & $\begin{array}{c}\text { 2. Llenar hasta la } \\
\text { estría del recipiente }\end{array}$ & $\begin{array}{c}50 \\
\%\end{array}$ & $\begin{array}{c}33.3 \\
\%\end{array}$ & $50 \%$ & 44.44 \\
\hline
\end{tabular}

$C=$ campesinos; $I=$ indígenas; $H A=$ Hijos de asalariados

Fuente: Elaboración propia

A partir del análisis de las tablas de consolidados y la confrontación de los argumentos que daban las niñas y los niños para cada situación se plantearon algunas hipótesis sobre la posible influencia del contexto social, como por ejemplo:

Cuando se les pregunta a los niños ¿por qué no llenar la botella?, ellos argumentaron que en el comercio las gaseosas nunca están completamente llenas. Es de anotar que una de las características encontradas a partir de observaciones hechas hasta el momento, es que la mayoría de los recipientes vistos en el mercado, no se encuentran completamente llenos. En el rastreo hecho a diferentes tiendas y supermercados se observó que la mayoría de los recipientes que contienen líquidos, el nivel de llenado va hasta donde la botella se curva o hasta la rosca. En particular, las botellas con agua y las saborizadas están llenas hasta la rosca y las que contienen gas, están proporcionales a la cantidad de gas. 
Otra justificación más frecuente que los niños expusieron para no llenar el recipiente en su totalidad, fue el problema de la movilidad del recipiente, ya que al llenarlos hasta el borde se podría derramar el líquido cuando se transporta de un sitio a otro. La actividad de transporte de agua es de gran importancia para las niñas y los niños de esta región, la cual sufre de la falta de este líquido.

\section{Conclusiones}

- La elaboración de las situaciones problema exigió la construcción de la definición del concepto de capacidad y permitió establecer que, para que dicho concepto se estructure es necesario tener conceptos tales como recipientes, volumen y contenencia.

- La capacidad como magnitud ha sido relegada por ser considerada de fácil manejo, por ello los textos solo hablan de la unidad (los litros) y en otros casos la consideran sinónimo del volumen. La solución de las situaciones problema evidenciaron que este concepto aún no estaba estructurado. En este trabajo se identificó que la capacidad requiere de esquemas de contenencia, de volumen y de recipientes, elementos que los niños aún no tienen estructurados y por tanto no pueden conceptualizar.

- Las estrategias evidencian una relación marcada que se tiene con el tamaño; es de anotar que según Piaget \& Szeminska (1967) esto es superado a los 8 años de edad. Lo anterior induce a la realización de un trabajo de refuerzo a la población en estudio, que les permita reconocer otras características de los recipientes para poder hacer comparaciones y establecer orden.

- Una de las características encontradas, a partir de observaciones hechas en el comercio, es que la mayoría de los recipientes no se encuentran completamente llenos. Esto se ha convertido en un referente para las niñas y los niños, ya que el mismo comercio marca el máximo nivel de contendido del recipiente. En las estrategias se evidencia la preferencia que el niño tiene frente al concepto de capacidad que maneja la actividad comercial, que a la definición matemática.
- El aula de clase, por todos los cambios sociales que en la actualidad se presentan, tiene diversidad cultural; tener en cuenta esto, implica establecer que cada grupo funciona con lógicas distintas, que en muchos casos pueden ser un obstáculo para que se comprendan los objetos matemáticos. Una tarea del docente, por ende, será establecer estas lógicas de su grupo de trabajo y generar puentes para que los niños alcancen el conocimiento deseado.

\section{Referencias}

Calambás, E. R. (2011). El concepto de capacidad en niños: un estudio de las estrategias escolares para su construcción. (Tesis de maestría). Universidad del Cauca, Popayán.

Chamorro, M. d. (2003). Didáctica de las matemáticas para primaria. Madrid, España: Pearson Prentice Hall.

González, M. J., \& Florez, P. (2001). Conocimiento profesional del profesor de secundaria sobre las matemáticas: el caso del volumen. Recuperado http://www.ugr.es/ pflores/textos/aRTICULOS/Propuestas/volumenart.pdf

Inhelder, B. (1978). Las estrategias Cognitivas: Aproximación al estudio de los procedimientos de resolución de problemas. Anuario de Psicología (18), 3-20.

Piaget, J., \& Szeminska, A. (1967). Las composiciones aditivas y multiplicativas de las relaciones y la igualdad de las diferencias. En J. Piaget, \& A. Szeminska, Génesis del número en el niño (pp. 261-288). Buenos Aires: Guadalupe.

Poussin, G. (2005). La entrevista Clínica. (N. P. Ayala, Trad.) México, México: Limusa S. A.

Vergnaud, G. (1982). Cognitive and developmental psychology and research in mathematics education: some theorical and metodological issues. For the Learning of Mathematics, 3(2), 31-41.

Vergnaud, G. (1990). La théorie des champs conceptuels. Recherches en Didáctique des Mathématiques, 10(2, 3), 133-170.

Vergnaud, G. (2006). Representación y actividad: dos conceptos estrechamente asociados. Teoría de la Representación y Educación Matemática, (p. 16). Madrid. 